Saint Louis University School of Law

Scholarship Commons

All Faculty Scholarship

2016

\title{
Collecting New Data on Disability Health Inequities
}

Elizabeth Pendo

Saint Louis University School of Law

Follow this and additional works at: https://scholarship.law.slu.edu/faculty

Part of the Health Law and Policy Commons

\section{Recommended Citation}

Pendo, Elizabeth, "Collecting New Data on Disability Health Inequities" (2016). All Faculty Scholarship. 50.

https://scholarship.law.slu.edu/faculty/50

This Article is brought to you for free and open access by Scholarship Commons. It has been accepted for inclusion in All Faculty Scholarship by an authorized administrator of Scholarship Commons. For more information, please contact erika.cohn@slu.edu, ingah.daviscrawford@slu.edu. 


\section{polioy and politios}

\section{Collecting New Data on Disability Health Inequities}

by Elizabeth Pendo

$\longrightarrow$ ne of the goals of The Patient Protection and Affordable Care Act is the reduction and elimination of health inequities, generally defined as population-level health differences that adversely affect disadvantaged groups. ${ }^{1}$ The ACA provides powerful new tools to collect, analyze, and share standardized data on these inequities. Prior to the ACA, disability was marginalized in data collection efforts, limiting our ability to understand and address significant health inequities experienced by millions of Americans. Now, for the first time, we can use these tools to collect valuable new data on the nature and extent of health inequities experienced by people with disabilities across the country.

Standardized health data collection is critical to health equity. The U.S. Department of Health and Human Services is a primary source of health and demographic data, and its data collection programs form the basis of reports such as the Healthy People series, which set out specific and measurable ten-year goals for nationwide health promotion, disease prevention, and elimination of health inequities, and The National Healthcare Quality and Disparities Report, the annual report of the Agency for Healthcare Research and Quality. The data collected by HHS programs is essential to identify inequities and to develop and assess programs to reduce health disparities. Prior to the ACA standards, inconsistent or incomplete data limited our ability to track the

March-April 2016 status of health and health care within or for disadvantaged populations. ${ }^{2}$

Standardized collection of disability data is particularly important because disability is not adequately captured through surveys or through medical and reimbursement records. HHS surveys have used inconsistent definitions of disability, targeted different populations, and omitted important questions about physical, programmatic, or attitudinal barriers. Disability is not consistently captured in medical and reimbursement records because a patient's impairment or disability may not be apparent to the clinician or raised by the patient as relevant to the clinical encounter. Although a diagnosis is more likely to be consistently captured in medical records, use of diagnoses to define the group would limit researchers and policy-makers to a reductive medical definition of disability, rather than capturing the broader barriers and disadvantages that need to be central concerns in efforts to eliminate health inequities. Finally, medical records may be underinclusive because individuals with disabilities never reach the clinical encounter: a recent study in five major cities found that men and women with mobility disabilities have difficulty simply making health care appointments. $^{3}$

Section 4302 of the ACA, "Understanding Health Disparities: Data Collection and Analysis," requires that all reporting from the secretary of HHS on federally conducted or supported health care or public health programs include separate data on race, ethnicity, sex, primary language, and disability status. Section 4302 also requires collection of disability-specific data regarding the barriers to health care experienced by people with disabilities. It directs HHS to identify locations where individuals with disabilities access different types of care and to determine the number of providers with accessible facilities and accessible medical and diagnostic equipment and the number of employees trained in disability awareness and in caring for patients with disabilities.

The ACA's inclusion of disability in HHS data collection standards is groundbreaking. Prior to the ACA, major reports such as Healthy People 2020 and The National Healthcare Quality and Disparities Report contained some disability health data, but coverage was limited because HHS data collection projects did not fully or consistently collect disability health information. ${ }^{4}$ Despite this, we know from numerous studies that people with disabilities face multiple barriers to care, use fewer preventive services, have poorer overall health outcomes, experience more preventable emergency department visits, and report more unmet needs and more dissatisfaction with the services they do receive. ${ }^{5}$ My research reveals that men and women with mobility disabilities encounter barriers in health care offices and facilities, including a lack of accessible medical and diagnostic equipment and a lack of policies or procedures designed to accommodate their needs and promote access. These physical and programmatic barriers are reinforced by attitudinal barriers such as assumptions, biases, or lack of knowledge about living with disability and lack of awareness of the federal laws that protect and promote accessible health care. ${ }^{6}$

While these studies and reports identify serious disability health inequities, we do not have the type of consistent and comprehensive data needed to 
identify, track, and address disability health inequities. We also lack disability-specific information, including comparisons between different types of disabilities or levels of severity of disability. In addition, although studies and legal actions under the Americans with Disabilities Act are suggestive, we do not know where people with disabilities access health care across the country or the nature and extent of physical, programmatic, or attitudinal barriers they encounter in those offices and facilities. These are critical gaps; according to recent U.S. census data one in five Americans lives with a disability, and many, if not most, of us will experience some type of impairment or disability over our lifespan. The ACA's inclusion of disability status and call for disability-specific information promises to remedy these gaps in our knowledge.

Health inequities have been a focus of public health efforts in the United States for over one hundred years, and the disproportionate burden of illness, suffering, and premature death experienced by already disadvantaged groups has been rightly recognized as a form of discrimination and social injustice. Health inequities are also an increasing concern within the health care system because they signal a pattern of lapses in quality and create excess cost. ${ }^{7}$ Why has it taken so long to acknowledge health inequities experienced by people with disabilities? While the reasons are complex and deeply rooted, one barrier is the lack of a consistent definition or a clear understanding of disability, which is a persistent problem across many areas of disability law, health law and policy, and bioethics.

In order to understand and address disability-linked health inequities, we must first understand disability itself. The challenge is to measure something that we do not fully understand, or understand in the same way. For example, the traditional medical model of disability defines disability as an individual, biological, and undesirable condition that requires medical treatment, while the social model of disability acknowledges that many of the disadvantages of disability are socially constructed and require changes to attitudes and environments. Although these models are not mutually exclusive, overemphasis on the personal, medical aspects of disability can obscure the socially constructed ones. Using an example from my research, consider a woman who uses a wheelchair and cannot have a mammogram because the imaging equipment requires her to stand and remain still for a series of images. The medical model suggests that her impairment and use of the wheelchair are the reasons she cannot access the needed care. The social model suggests that the socially created barriers, including lack of accessible medical and imaging equipment and of policies and procedures to accommodate patients who cannot stand or remain still, are the reasons.

In October 2011, HHS issued its final data collection standards related to disability, race, ethnicity, and primary language. ${ }^{8}$ The standards require the use of, at a minimum, the same six questions used by the American Community Survey and other major surveys to gauge disability among the U.S. population. The questions cover six disability categories-hearing, vision, cognitive, ambulatory, self-care, and independent living difficulties - and are aimed at function, rather than diagnosis. There are some concerns that the six categories are not sufficiently inclusive and that they focus on a medical or individual measure of disability. However, use of a standard and proven set of questions across all federally conducted surveys and health plans is a step forward and could provide a basis for collection of additional disability-specific data regarding social or environmental barriers to health care.

The ACA's inclusion of disability status as a health inequity demographic and call for disability-specific health care information holds great promise for new, standardized data on the nature and extent of a wide range of inequities experienced by people with disabilities. It also signals a much-needed move toward explicit and meaningful inclusion of people with disabilities in efforts to structure our health care system to provide effective, high-quality care for all.

1. U.S. Department of Health and Human Services, Secretary's Advisory Committee on National Health Promotion and Disease Prevention Objectives for 2020, Phase I report: "Recommendations for the Framework and Format of Healthy People 2020," section IV: "Advisory Committee findings and recommendations," accessed February 3, 2016, at http://www.healthypeople.gov/2020/about/ advisory/Reports.

2. R. Dorsey, G. Graham, S. Glied, et al., "Implementing Health Reform: Improved Data Collection and the Monitoring of Health Disparities," Annual Review of Public Health 35 (2014): 123-38.

3. T. Lagu, N. S. Hannon, M. B. Rothberg, et al., "Access to Subspecialty Care for Patients with Mobility Impairments: A Survey," 158, no. 6 Annals of Internal Medicine (2013): 443-44.

4. L. I. Iezzoni, "Tracking Disability Disparities: The Data Dilemma," Journal of Health Services Research \& Policy 13, no. 3 (2008): 129-30.

5. National Council on Disability, The Current State of Health Care for People with Disabilities (summarizing findings), 2009, accessed February 3, 2016, https://www.ncd. gov/publications/2009/Sept302009; see p. 10.

6. E. Pendo, "Disability, Equipment Barriers and Women's Health: Using the ADA to Provide Meaningful Access," St. Louis University Journal of Health Law \& Policy 2, no. 1 (2008): 15-56; E. Pendo, "Reducing Disparities through Health Care Reform: Disability and Accessible Medical Equipment," Utah Law Review 4 (2010): 1057-83.

7. T. A. LaVeist, D. J. Gaskin, and P. Richard, The Economic Burden of Health Inequities in the United States (Washington, D.C.: Joint Center for Political and Economic Studies, 2009).

8. Dorsey et al., "Implementing Health Reform."

DOI: $10.1002 /$ hast. 540

This column appears by arrangement with the American Society for Bioethics and Humanities. 